\title{
Transrectal versus transperineal 14-core prostate biopsy in detection of prostate cancer: A comparative evaluation at the same Institution
}

\author{
Maria Angela Cerruto ${ }^{1}$, Fabio Vianello ${ }^{2}$, Carolina D'Elia ${ }^{1}$, Walter Artibani ${ }^{1}$, Giovanni Novella ${ }^{1}$ \\ ${ }^{1}$ Department of Surgery, Urology Clinic, AOUI Verona, Italy; \\ ${ }^{2}$ Urology Clinic, University of Padua, Italy.
}

\begin{abstract}
Summary Background: The ideal bioptic strategy
for CaP detection is still to be completely defined. The aim of our study is to compare transperineal (TP) and transrectal (TR) approaches, in a 14-core initial prostate biopsy for CaP detection.

Material and methods: A prospective controlled study was conducted enrolling 108 consecutive patients with a PSA level greater than $4 \mathrm{ng} / \mathrm{mL}$ and/or an abnormal DRE. TR versus TP 14-core initial prostatic biopsies were performed on 54 and 54 patients, respectively, with a randomisation ratio of 1:1.
\end{abstract}

Results: The cancer detection rates were 46.29 (25 out of 54 patients), and $44.44 \%$ (24 out of 54 patients), respectively, using the TR or the TP approach $(p=0.846)$. The overall cancer core rate was significantly higher when the TP approach was used: 21.43\% (162 out of 756 cores) and $16.79 \%$ (127 out of 756 cores), with the TP and the TR approach, respectively $(p=0.022)$. The cores were signifcantly longer performing TP approach: at the site "1" (14.92 versus $12.97 \mathrm{~mm}, p=0.02)$; at " 5 " (15.53 versus $13.69 \mathrm{~mm}, p=0.037$ ); at " 7 " (15.06 versus $12.86 \mathrm{~mm}$, $p=0.001)$; at "9" (14.92 versus $13.38 \mathrm{~mm}, p=0.038)$; at "11" (16.32 versus $12.31 \mathrm{~mm}, p=0.0001)$; at "12" (15.14 versus $12.19 \mathrm{~mm}, p=0.0001$ ); at "13" (17.49 versus $13.98 \mathrm{~mm}, p=0.0001$ ); at " 14 " (16.77 versus 13.36 $\mathrm{mm}, p=0.0001$ ). As to the biopsy related pain, the mean pain level perceived by patients during the TR approach was $1.56 \pm 1.73$ versus $1.42 \pm 1.37$ registered during TP approach $(p=0.591)$.

Conclusions: No significant differences were found in cancer detection rate, cancer core rate between TP and TR approaches for prostatic biopsy. Even in terms of complication rate or pain level, it cannot be concluded that one procedure is superior to the other one. Apparently, strictly following our protocol, TP approach seems to offer a better sampling at the level of the apex and the $T Z$, however without adding any significant advantage in terms of overall cancer detection rate.

KEY WORDS: Prostatic neoplasm; Transrectal biopsy;

Transperineal biopsy; Diagnosis; Cancer detection rate; Prostatespecific antigen.

Submitted 12 February 2014; Accepted 30 June 2014

\section{INTRODUCTION}

The widespread use of serum prostate specific antigen (PSA) measurement as an opportunistic screening tool to detect early prostate cancer (CaP) led to optimise an effective biopsy technique. Unfortunately, the ideal bioptic strategy for $\mathrm{CaP}$ detection is still to be completely defined. At the present, there is still a lack of standardisation regarding both transperineal (TP) and transrectal (TR) approaches.

In 2003, Emiliozzi et al. carried out a prospective study aiming of comparing the efficacy of TP and TR six-core prostatic biopsy. They performed both biopsy approaches, on the same patients, stating that TP biopsy resulted superior to TR one to detect $\mathrm{CaP}(\mathrm{p}=0.012)(1)$.

In 2007, firstly, Kawakami et al. demonstrated that an extended TP biopsy was as effective as its TR counterpart in detecting the presence and the characteristics of the $\mathrm{CaP}$, as far as sampling sites were selected to maximize the cancer detection rate (2).

More recently, Hara et al. carried out a prospective randomized study comparing TP with TR 12-core biopsy, in 246 patients with PSA levels ranging from 4.0 to 20.0 $\mathrm{ng} / \mathrm{mL}$ (3). With patients in lithotomy position, all procedures were performed using spinal anaesthesia $(0.5 \%$ bupivacaine) or a caudal block (1\% lidocaine) according to TP and TR approaches, respectively. The authors did not find any significant difference in cancer detection rate, cancer core rate or complications between the two approaches: they concluded that the preferred approach, as the initial prostate biopsy, might be the TR one as it did not require spinal anaesthesia or other burdensome related processes (3). Nevertheless, the same study group even reported that, with a PSA in the so-called "grey zone", significantly more cores were positive when the TP approach was applied, especially for cores coming from the transition zone (4). Conclusions were that urologist's preference could be sufficient for choosing the ideal approach, except for a possible small advantage for TP biopsy when PSA is in the "grey zone".

Just to compare TP and TR approaches, in a 14-core initial prostate biopsy for $\mathrm{CaP}$ detection, we have performed a prospective controlled study and these results are herein presented. 


\section{Material And Methods}

A prospective controlled study was conducted enrolling 108 consecutive patients at our urological Center with a PSA level greater than $4 \mathrm{ng} / \mathrm{mL}$ and/or an abnormal digital rectal examination (DRE). TR versus TP 14-core initial prostatic biopsies were performed on 54 and 54 patients, respectively, with a randomisation ratio of $1: 1$. The inclusion criteria foresaw no previous prostate biopsy, no history of $\mathrm{CaP}$ and no clinical evidence of acute or chronic prostatitis. All patients were adequately informed on the execution modalities of the bioptic procedure and on its potential complications. They were asked to provide a written consent. Each patient underwent a clinical evaluation that included DRE and transrectal ultrasound (TRUS). Prostate volume (PV) was measured by means of TRUS and was calculated as the height per the width per the length per 0.52. Table 1 lists patients' characteristics: no significant differences were found in background factors between the two groups.

All patients were instructed to discontinue an eventual anticoagulant therapy for at least 7 days before and after the prostate biopsy. All patients were given an enema the same morning of the procedure and an antibiotic coverage was provided in all cases using an oral fluoroquinolone (prulifloxacin, $600 \mathrm{mg}$, once a day) for 3 days, starting from the day before the biopsy. For both approaches, the patients were placed in lithotomy position, and all biopsies were carried out only by two skilled urologists included as the co-authors of this paper: GN for the TP approach, and FV for the TR one.

All the TP biopsies were performed using a single median TP access $1.5 \mathrm{~cm}$ above the anal sphincter, as previously described (5). In all cases, local anaesthesia was provided releasing $2 \mathrm{~mL}$ of $1 \%$ mepivacaine at the level of the prostate apex. A 18-gauge coaxial needle (TruGuide Bard, $13 \mathrm{~cm}$ long) was inserted up to the prostate apex through the anesthetised perineal path under TRUS guidance. On the removal of the blunt tip stylet, the guiding cannula of the coaxial needle was used as a TP metallic path for repeated atraumatic passages of the biopsy needle.

With the TP approach, firstly, a traditional sextant biopsy was performed; then, additional lateral sextant peripheral cores were added and, lastly, two cores were taken from the anterior transitional zone (TZ) (6).

For systematic TR biopsy, a bilateral periprostatic nerve block was obtained using a 1\% lidocaine solution, transrectally injected under ultrasound guidance at the prostate apex and the seminal vesicle-prostatic angles. Eight cores were added to the standard TR protocol described by Hodge et al (7): six, far laterally in the peripheral zone (PZ), and two in the middle TZ.

TP and TR approaches were both performed under TRUS guidance (Siemens Sonoline Omnia Diagnostic Ultrasound System with a 7.75-MHz linear probe was used). With both approaches, an 18-gauge Tru-Cut needle with a cutting length of $23 \mathrm{~mm}$ was applied to obtain specimens.

Overall 14-core TRUS guided prostate biopsies have been obtained: 12 specimens from PZ and two from TZ for each approach. Cores of the standard sextant were conventionally labelled from 1 to 6 . Likewise, additional peripheral cores from the lateral part of prostatic apex, were numbered as "7" and "8". Additional lateral peripheral cores, from the mid prostate, were numbered as " 9 " and "10". Other cores, from the anterior horn, were numbered as "11" and "12". Finally, biopsies taken from the TZ were labelled as " 13 " and "14".

In all cases, pain level during the bioptic procedure, was evaluated by means of a visual analogue scale/numeric analogue scale in which 0 corresponded to "no pain" and 10 to "the worst, imaginable pain" $(5,6)$. All patients were clinically evaluated 30 days after the biopsy to record eventual complications related to procedures (5).

We determined the CaP detection rate, the cancer core rate (ratio of the number of cancer-positive cores to the total number of biopsy specimens) and any complications occurred in order to define efficacy and tolerability of the TP biopsy compared with the TR one.

For statistical analysis, chi-square and the MannWhitney $\mathrm{U}$ tests were used and a $\mathrm{p}<0.05$ was considered significant.

\section{RESULTS}

Patients' characteristics are listed in Table 1. The cancer detection rates were 46.29 (25 out of 54 patients), and $44.44 \%$ (24 out of 54 patients), respectively, using the TR or the TP approach $(\mathrm{p}=0.846)$. Among patients with PSA levels of less than $10.0 \mathrm{ng} / \mathrm{mL}$, the detection rate was 42.22

\section{Table 1.}

Patients' characteristics.

\begin{tabular}{|c|c|c|c|}
\hline Characteristics & TP approach & TR approach & $P$ value \\
\hline Patients (n) & 54 & 54 & NS \\
\hline Mean age (year) (SD) & $66.50 \pm 8.87$ & $67.30 \pm 8.05$ & 0.627 \\
\hline Mean PSA (ng/mL) (SD) & $15.95 \pm 41.04$ & $12.36 \pm 39.65$ & 0.646 \\
\hline $\mathrm{BMI}\left(\mathrm{Kg} / \mathrm{cm}^{2}\right)(\mathrm{SD})$ & $27.16 \pm 3.18$ & $27.00 \pm 3.12$ & 0.794 \\
\hline $\begin{array}{l}\text { Mean prostate volume } \\
\left(\mathrm{cm}^{3}\right)(\mathrm{SD})\end{array}$ & $56.29 \pm 31.33$ & $61.49 \pm 33.39$ & 0.408 \\
\hline Abnormal DRE & $11 / 54(20.37)$ & $10 / 54(18.52 \%)$ & 0.810 \\
\hline
\end{tabular}

Table 2.

Comparison of cancer detection rate according to PSA level and prostate volume (determined by TRUS), between TP and TR approach.

\begin{tabular}{|l|c|c|c|}
\hline Variables & TP approach (\%) & TR approach (\%) & P value \\
\hline Overall & $24 / 54(44.44)$ & $25 / 54(46.29)$ & $0.846(\mathrm{NS})$ \\
\hline PSA $(\mathrm{ng}(\mathrm{mL})$ & & & $0.303(\mathrm{NS})$ \\
\hline$\leq 10$ & $15 / 39(38.46)$ & $19 / 45(42.22)$ & \\
\hline$>10.1$ & $9 / 15(60.00)$ & $6 / 9(66.67)$ & \\
\hline Prostate volume $\left(\mathrm{cm}^{3}\right)$ & & & $0.283(\mathrm{NS})$ \\
\hline$<30$ & $8 / 9(88.89)$ & $8 / 10(80)$ & \\
\hline $30-50$ & $11 / 20(55.00)$ & $7 / 13(53.85)$ & \\
\hline$>50$ & $5 / 25(20.00)$ & $10 / 30(33.33)$ & \\
\hline NS $=$ Not Significant. & & & \\
\hline
\end{tabular}


Table 3.

Comparison of cancer-positive core rate by anatomic location between transperineal and transrectal approaches

\begin{tabular}{|l|c|c|c|c|c|c|}
\hline Approach & Total & PZ & TZ & Apex & Mid prostate & Base \\
\hline TP & 756 & 648 & 108 & 216 & 216 & 216 \\
& 162 & 140 & 22 & 49 & 48 & 46 \\
& $21.43 \%$ & $21.60 \%$ & $20.37 \%$ & $22.68 \%$ & $22.22 \%$ & $21.30 \%$ \\
\hline TR & 756 & 648 & 108 & 216 & 216 & 216 \\
Biopsy cores (n) & 127 & 114 & 13 & 38 & 40 & 44 \\
Cancer cores (n) & $16.7 \%$ & $17.59 \%$ & $12.04 \%$ & $17.59 \%$ & $18.52 \%$ & $20.47 \%$ \\
Cancer core rate (\%) & 0.022 & 0.068 & 0.097 & 0.186 & 0.340 & 0.814 \\
P value & $(\mathrm{NS})$ & $(\mathrm{NS})$ & $(\mathrm{NS})$ & $(\mathrm{NS})$ & (NS) \\
\hline NS = Not Significant. & & & & & \\
\hline
\end{tabular}

\section{Discussion}

To our knowledge, the present study is the first prospective controlled evaluation that compares systematic 14-core biopsy using TR and TP approaches, both under local anaesthesia. In 2003, Emiliozzi et al. reported a comparison between the two approaches, using the same patients, under local anaesthesia. The aim of that study was to compare the efficacy of TP versus TR six-core prostate biopsies, performing six TP plus six TR biopsies in a group of 107 patients with PSA greater than $4 \mathrm{ng} / \mathrm{mL}$.

The authors highlighted the superiority of the TP approach with a cancer detection rate of $40 \%$ (43 out of 107) using the combination of both

Table 4.

Complication rates.

\begin{tabular}{|l|c|c|c|}
\hline Characteristics & TP approach & TR approach & P value \\
\hline Overall (\%) & $7 / 54(12.96)$ & $7 / 54(12.96)$ & NS \\
\hline Rectal bleeding (\%) & $0(0)$ & $4(57.16)$ & 0.04 \\
\hline Urinary retention (\%) & $0(0)$ & $1(14.28)$ & $0.315(\mathrm{NS})$ \\
\hline Urethral bleeding (\%) & $5(71.43)$ & $0(0)$ & 0.022 \\
\hline Vasovagal event (\%) & $2(28.57)$ & $1(14.28)$ & $0.56(\mathrm{NS})$ \\
\hline Fever $>38.5^{\circ} \mathrm{C}(\%)$ & $0(0)$ & $1(14.28)$ & $0.315(\mathrm{NS})$ \\
\hline Not Significant. & & \\
\hline
\end{tabular}

(19 out of 45 ) and $38.46 \%$ (15 out of 39) when the TR or the TP approach was applied, respectively $(\mathrm{p}=0.728)$.

Stratifying patients for either PSA level or PV, no significant differences were found in the two groups (Table 2). The overall cancer core rate was significantly higher when the TP approach was used: $21.43 \%$ (162 out of 756 cores) and $16.79 \%$ (127 out of 756 cores), with the TP and the TR approach, respectively $(\mathrm{p}=0.022)$. The cancer core rate, in PZ cores, was $17.59 \%$ (114 out of 648 cores) in case of TR approach, and $21.43 \%$ (140 out of 648 cores $)$ going transperineally $(p=0.068)$. In $T Z$, the corresponding rate was 12.04 (13 out of 108 cores) versus $20.37 \%$ (22 out of 108 cores), respectively ( $\mathrm{p}=$ 0.097) (Table 3).

The cores were significantly longer performing TP approach, as it follows: at the site "1" (14.92 versus 12.97 $\mathrm{mm}, \mathrm{p}=0.02)$; at " 5 " (15.53 versus $13.69 \mathrm{~mm}, \mathrm{p}=0.037$ ); at "7" (15.06 versus $12.86 \mathrm{~mm}, \mathrm{p}=0.001)$; at "9" (14.92 versus $13.38 \mathrm{~mm}, \mathrm{p}=0.038$ ); at " 11 " (16.32 versus 12.31 $\mathrm{mm}, \mathrm{p}=0.0001)$; at "12" (15.14 versus $12.19 \mathrm{~mm}$, $\mathrm{p}=0.0001)$; at "13" (17.49 versus $13.98 \mathrm{~mm}, \mathrm{p}=0.0001)$; at "14" (16.77 versus $13.36 \mathrm{~mm}, \mathrm{p}=0.0001)$. Overall, no significant differences were found in terms of post-biopsy complications between the two groups (Table 4).

As to the biopsy related pain, the mean pain level perceived by patients during the TR approach was $1.56 \pm$ 1.73 versus $1.42 \pm 1.37$ registered during TP approach $(\mathrm{p}=0.591)$. approaches; of 38\% (41 of 107) with the TP approach alone, and 32\% (34 of 107) when the TR approach had been applied alone (1).

More recently, Hara et al prospectively compared TP and TR approaches and they did not show any significant differences in overall cancer detection rate (3). As to the suggested superiority of the TP approach in detecting TZ cancer, Shannon et al. reported that the TP approach was more successful in detecting TZ cancer because the correct diagnosis rate was greater when the TP approach was used in comparison with the TR approach (89 versus 68\%)(8). Furthermore, Furuno et al., performing a TP ultrasound-guided template biopsy in men with PSA levels ranging between 4 and $10 \mathrm{ng} / \mathrm{mL}$, reported that the cancer core rate of the biopsies from the anterior part of the prostate was significantly greater than that from the posterior region (9).

They suggested that TR sextant biopsy might be inadequate for detecting cancer localized in the anterior region. On the contrary, Hara et al. did not find any differences in cancer core rates whatever zones or approaches were (3).

In authors' opinion, the increased number of biopsy specimens to 12 might reduce the differences in cancer detection rates between the two approaches. In our study, cancer core rates in $\mathrm{PZ}, \mathrm{TZ}$, apex and mid prostate were always higher when TP approach was used, but without reaching any statistical significance. More recently, Takenada, Hara et al. found that, in patients with PSA in the "grey zone" (ranging between 4.1 and $10.0 \mathrm{ng} / \mathrm{mL}$ ), significantly more cores were positive when TP approach was applied, especially regarding to TZ cores (4).

They concluded that urologists' preferences should be sufficient for choosing the best approach, except for possible small advantages for TP biopsy when PSA is in the "grey zone".

The results of our study might even support this trend towards a possible advantage of TP biopsy in better sampling both TZ and prostate apex. Some of our group had previously reported that TP approach would allow a greater sampling of the prostate apex compared with midgland and prostate base $(\mathrm{p}<0.001)(10)$. To possibly 
confirm this statement, in the present series, the cores resulted significantly longer with the TP rather than with the TR approach, mainly at the apex and in the TZ. However, the real advantage of these data is still uncertain as these findings are supported by no significant differences in terms of cancer detection rate reached when the two approaches are used.

As to the adverse events, no differences were found in the overall incidence of complications as a result occurring with the two approaches, except for the urethral bleeding in TP group, and the rectal bleeding in TR group.

As to some technical difficulties, many authors stated the TR approach is a by far easier procedure and patients' discomfort may be prevented, using only local anaesthesia. Moreover, the TP approach may be not familiar to the majority of the urologists and many patients may complain of some pain when only local anaesthesia is used.

However, in our hands, both approaches showed a similar, small, and acceptable discomfort.

In our opinion, both methods should be or become equally familiar to urologists.

\section{Conclusions}

Our results confirmed no significant differences were found in cancer detection rate, cancer core rate between TP and TR approaches for prostatic biopsy. Even in terms of complication rate or pain level, it cannot be concluded that one procedure is superior to the other one. Apparently, strictly following our protocol, TP approach seems to offer a better sampling at the level of the apex and the TZ, however without adding any significant advantage in terms of overall cancer detection rate.

\section{REFERENCES}

1. Emiliozzi P, Corsetti A, Tassi B, et al. Best approach for prostate cancer detection: a prospective study on transperineal versus transrectal six-core prostate biopsy. Urology. 2003; 61:961-6.

2. Kawakami S, Yamamoto S, Numao N, et al. Direct comparison between transrectal and transperineal extended prostate biopsy for detection of cancer. Int J Urol. 2007; 14:719-24.

3. Hara R, Jo Y, Fuji T, et al: Optimal approach for prostate cancer detection as initial biopsy: prospective randomized study comparing transperineal versus transrectal systematic 12-core biopsy. Urology. 2008; 71:191-5.

4. Takenaka A, Hara R, Ishimura $T$, et al. A prospective randomized comparison of diagnostic efficacy between transperineal and transrectal12-core prostate biopsy. Prostate Cancer Prostatic Dis. 2008; 11:134-8.

5. Novella G, Ficarra V, Galfano A, et al. Pain assessment after original transperineal prostate biopsy using a coaxial needle. Urology. 2003; 62:689-92.

6. Ficarra V, Novella G, Novara G et al. The potential impact of prostate volume in the planning of optimal number of cores in the systematic transperineal prostate biopsy. Eur Urol. 2005; 48:932-7.

7. Hodge KK, McNeal JE, Terris MK, et al: Random systematic versus directed ultrasound guided transrectal core biopsies of the prostate. J Urol. 1989; 142:71-5.

8. Shannon BA, McNeal JE, Cohen RJ. Transitional zone carcinoma of the prostate gland: a common indolent tumor type that occasionally manifests aggressive behaviour. Pathology. 2003; 35:467-71.

9. Furuno T, Demura T, Kaneta $T$, et al. Difference of cancer core distribution between first and repeat biopsy in patients diagnosed by extensive transperineal ultrasound guided template prostate biopsy. Prostate. 2004; 58:76-81.

10. Ficarra V, Martignoni $G$, Novella $G$, et al. Needle core length is a quality indicator of systematic transperineal prostate biopsy. Eur Urol. 2006; 50:266-71.

\author{
Correspondence \\ Maria Angela Cerruto, MD \\ mariaangela.cerruto@univr.it \\ Carolina D'Elia, MD, FEBU (Corresponding Author) \\ karolinedelia@gmail.com \\ Walter Artibani, MD \\ walter.artibani@univr.it \\ Giovanni Novella, MD \\ giovanni.novella@ospedaleuniverona.ir \\ Urology Clinic, AOUI Verona \\ Piazzale L. Scuro 10 - 37134 Verona, Italy \\ Fabio Vianello, MD \\ fabio.vianello@unipd.it \\ Urology Clinic, University of Padua, Padova, Italy
}

